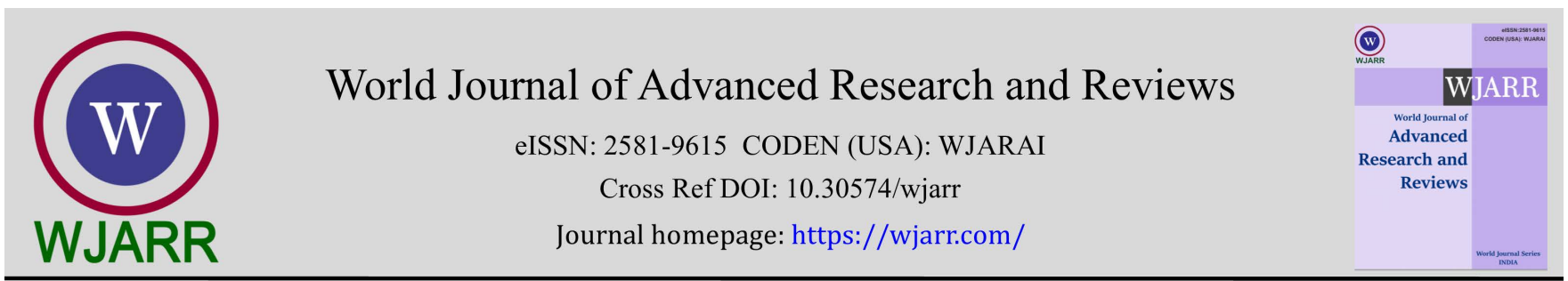

(RESEARCH ARTiClE)

\title{
Determination of time evolution of the gravitational constant in the framework of Brans-Dicke Theory: An easy way
}

\author{
Sudipto Roy* \\ Department of Physics, St. Xavier's College, Kolkata, West Bengal, India.
}

World Journal of Advanced Research and Reviews, 2021, 11(02), 163-168

Publication history: Received on 08 July 2021; revised on 12 August 2021; accepted on 14 August 2021

Article DOI: https://doi.org/10.30574/wjarr.2021.11.2.0375

\begin{abstract}
The present article demonstrates a very simple mathematical way to determine the time-dependence of the dynamical gravitational constant $(G)$ in the framework of the Brans-Dicke theory of gravity. Brans-Dicke field equations, for a matter-dominated, pressure-less and spatially flat universe with homogeneous and isotropic space-time, have been used for this formulation. The gravitational constant $(G)$ is the reciprocal of the Brans-Dicke scalar field $(\phi)$. Using a simple ansatz, which represents the Brans-Dicke scalar field $(\phi)$ as a function of time, the possible values of a constant parameter (constituting the ansatz) have been calculated with the help of the field equations, using the values of some cosmological parameters at the present time. The values of that parameter (belonging to the ansatz) lead to the conclusion that the scalar field $(\phi)$ decreases and consequently the gravitational constant $(G)$ increases with time. The value of the relative time-rate of change of the gravitational constant (i.e., $\dot{G} / G$ ) has also been estimated and this quantity has been found to be independent of time. Time-dependence of $\phi$ and $G$ has been depicted graphically for all values of the parameter belonging to the ansatz. The novel features of this study are that the gravitational field equations did not have to be solved, unlike other studies, to arrive at the results and the mathematical scheme for calculations is extremely easy in comparison to other recent studies in this regard.
\end{abstract}

Keywords: Cosmology; Brans-Dicke Theory; Scalar Field; Gravitational Constant

\section{Introduction}

Lots of exciting astrophysical observations and corresponding theoretical findings have motivated people to carry out research on alternative theories of general relativity. Several theories of modified gravity have been proposed for such explorations [1-6]. Among them, the $\mathrm{f}(\mathrm{R})$ theory and the Brans-Dicke (BD) theory are highly noteworthy [7-9]. In 1961, Brans and Dicke proposed a remarkably interesting theory of gravitation, based on Mach's principle [10, 11]. Its theoretical framework was so constructed that the gravitational constant $(G)$ could be obtained from the structure and dynamics of the universe. For this purpose, a scalar field parameter $(\phi)$ was introduced in such a way that $G$ behaves as $\phi^{-1}$. The time-varying nature of $G$ has been indicated by the findings of a number of recent observations [12-16]. BD theory accounts for several cosmological phenomena in terms of a time-varying $\phi[17,18]$. Using the original BD theory, it was difficult to explain astrophysical observations regarding the accelerated cosmic expansion [9, 19-21]. A new component, named dark energy, was introduced to explain the accelerated expansion of the universe and its change of phase from deceleration to acceleration [22].

In the present study, the nature of time-dependence of the gravitational constant $(G)$ has been determined with the help of Brans-Dicke field equations constructed for an isotropic and homogeneous space-time, in a matter-dominated universe with zero spatial curvature and zero pressure (dust filled). A simple ansatz, in the form of an exponential function of time, has been assumed here to represent the time-variation of the scalar field $(\phi)$. Incorporating this ansatz

\footnotetext{
* Corresponding author: Sudipto Roy; Email: roy.sudipto@sxccal.edu

Department of Physics, St. Xavier's College, Kolkata, West Bengal, India.
} 
into the field equations, we have found $\phi$ to be a decreasing function of time, using the values of some cosmological parameters at the present time (i.e., at $t=t_{0}$ ). Without solving the differential equations involving the scale factor and its time derivatives (i.e., the field equations of the BD theory), the characteristics of time evolution of $G(\equiv 1 / \phi)$ have been determined. The gravitational constant has been found to be increasing with time. The relative rate of change of the gravitational constant (i.e., $\dot{G} / G$ ) has been determined and it has been found to be independent of time.

\section{Brans-Dicke Field Equations}

For a matter-dominated, homogeneous and isotropic universe with zero spatial curvature and zero pressure (dust filled), Brans-Dicke field equations are given by,

$$
\begin{aligned}
& 3 \frac{\dot{a}^{2}}{a^{2}}+3 \frac{\dot{a} \dot{\phi}}{a \phi}-\frac{\omega \dot{\phi}^{2}}{2 \phi^{2}}=\frac{\rho_{m}}{\phi} \\
& 2 \frac{\ddot{a}}{a}+\frac{\dot{a}^{2}}{a^{2}}+\frac{\omega \dot{\phi}^{2}}{2 \phi^{2}}+2 \frac{\dot{a} \dot{\phi}}{a \phi}+\frac{\ddot{\phi}}{\phi}=0 \\
& \frac{\ddot{\phi}}{\phi}+3 \frac{\dot{a} \dot{\phi}}{a \phi}=\frac{\rho_{m}}{2 \omega+3} \frac{1}{\phi}-\frac{\dot{\omega}}{2 \omega+3} \frac{\dot{\phi}}{\phi}
\end{aligned}
$$

Here $a, \phi, \omega$ and $\rho_{m}$ are respectively the scale factor, scalar field, Brans-Dicke coupling parameter and the density of matter (dark matter + baryonic matter). The symbols $\dot{a}$ and $\ddot{a}$ stand for the first order and the second order derivatives, respectively, of the scale factor $(a)$ with respect to time. These symbols represent the same operations (i.e., $1^{\text {st }} \& 2^{\text {nd }}$ order differentiations with respect to time, respectively) when used for any other variable in the present study.

A combination of equations (1) and (2) lead to the following relation.

$$
4 \frac{\dot{a}^{2}}{a^{2}}+2 \frac{\ddot{a}}{a}=\frac{\rho_{m}}{\phi}-5 \frac{\dot{a}}{a} \frac{\dot{\phi}}{\phi}-\frac{\ddot{\phi}}{\phi}
$$

Using the definitions of the Hubble parameter $\left(H=\frac{\dot{a}}{a}\right)$ and deceleration parameter $\left(q=-\frac{\ddot{a} a}{\dot{a}^{2}}\right)$, equation (4) can be written as,

$$
4 H^{2}-2 q H^{2}=\frac{\rho_{m}}{\phi}-5 H \frac{\dot{\phi}}{\phi}-\frac{\ddot{\phi}}{\phi}
$$

According to Brans-Dicke theory, the gravitational constant $(G)$ and its relative time-rate of change $(\dot{G} / G)$ are given by,

$$
G=\frac{1}{\phi} \text { and } \frac{\dot{G}}{G}=-\frac{\dot{\phi}}{\phi}
$$

The values of cosmological parameters at the present time (i.e., at $t=t_{0}$ ), used in this article, are given below. Here $t_{0}$ stands for the age of the universe.

$$
\begin{aligned}
& H_{0}=2.39 \times 10^{-18} \mathrm{sec}^{-1}, \quad t_{0}=4.13 \times 10^{17} \mathrm{sec}, \quad \rho_{m_{0}}=2.97 \times 10^{-27} \mathrm{~kg} / \mathrm{m}^{3} \quad, \quad G_{0}=6.67 \times \\
& 10^{-11} \mathrm{~N} \mathrm{~m}^{2} \mathrm{Kg}^{-2}, \phi_{0}=1 / G_{0}=1.50 \times 10^{10} \mathrm{~N}^{-1} \mathrm{~m}^{-2} \mathrm{Kg}^{2}, q_{0}=-0.55
\end{aligned}
$$

\section{A Model for the Scalar Field $(\phi)$}

We assume the following ansatz to represent the time dependence of the scalar field $(\phi)$.

$$
\phi=\phi_{0} \operatorname{Exp}\left[m\left(t-t_{0}\right)\right]
$$


In equation (7), $m$ is a constant parameter having the dimension of $t i m e^{-1}$. Its value can be determined with the help of the field equations.

Based on equation (7) we obtain,

$$
\frac{\dot{\phi}}{\phi}=m \text { and } \frac{\ddot{\phi}}{\phi}=m^{2}
$$

Substituting the above relations into equation (5), we get,

$$
m^{2}+5 H m+2 H^{2}(2-q)-\frac{\rho_{m}}{\phi}=0
$$

Equation (9) is a relation among some time dependent cosmological parameters $\left(H, q, \phi, \rho_{m}\right)$. This relation must be valid at all instants of cosmic time (including the present time). Replacing these parameters by their values at the present time (i.e., at $t=t_{0}$ ) we get,

$$
m^{2}+5 H_{0} m+2 H_{0}^{2}\left(2-q_{0}\right)-\frac{\rho_{m_{0}}}{\phi_{0}}=0
$$

Equation (10) is quadratic in $m$. The roots of this quadratic equation are given by,

$$
m_{1,2}=\frac{-5 H_{0} \pm \sqrt{25 H_{0}{ }^{2}-8 H_{0}{ }^{2}\left(2-q_{0}\right)+4 \rho_{m_{0}} / \phi_{0}}}{2}
$$

In equation (11), the subscripts 1 and 2 of $m$ correspond to plus and minus signs respectively in the numerator of its expression. Using the values of cosmological parameters (mentioned in section 2), we get $m_{1}=-3.37 \times 10^{-18}$ and $m_{2}=-8.58 \times 10^{-18}$. The negative signs of these values clearly imply that $\phi$ decreases with time, as per equation (7). Thus, $G(\equiv 1 / \phi)$ increases with time. Using equation (7), the time-dependence of $G$ can be expressed as,

$$
G=\frac{1}{\phi}=\frac{1}{\phi_{0}} \operatorname{Exp}\left[-m\left(t-t_{0}\right)\right]
$$

Combining equation (6) with equation (8) we get, $\frac{\dot{G}}{G}=-\frac{\dot{\phi}}{\phi}=-m$. Thus, $\dot{G} / G$ is independent of time. Its possible values are, $\frac{\dot{G}}{G}=-m_{1}=3.37 \times 10^{-18} \mathrm{~s}^{-1}=1.06 \times 10^{-10} \mathrm{Yr}^{-1}$ and $\frac{\dot{G}}{G}=-m_{2}=8.58 \times 10^{-18} \mathrm{~s}^{-1}=2.70 \times 10^{-10} \mathrm{Yr}^{-1}$. These values are consistent with the upper limit (i.e., $4 \times 10^{-10} \mathrm{Yr}^{-1}$ ) for $\left|\frac{\dot{G}}{G}\right|_{0}$, as stipulated by Steven Weinberg [23].

\section{Results and discussion}

Figure 1 shows the variation of the scalar field $(\phi)$ as a function of time, based on equation (7), for two values of the parameter $m$ obtained from equation (11). Since both values of $m$ are negative, $\phi$ decreases with time in accordance with equation (7). Figure 2 shows the time evolution of the gravitational constant $(G)$, based on equation (12), for two values of the parameter $m$ obtained from equation (11). The gravitational constant $(G)$ is found here to be increasing with time due to the negative values of $m$. This nature of time variation of $G$ is in qualitative agreement with the findings of some recent studies based on models which are completely different from the present one [24-26]. Both $\phi$ and $G$ have been plotted here in logarithmic scales for a greater visual clarity of their values obtained from the graphs. Logarithm of $\phi$ and $G$ vary linearly with time, in accordance with equations (7) and (12) respectively. 


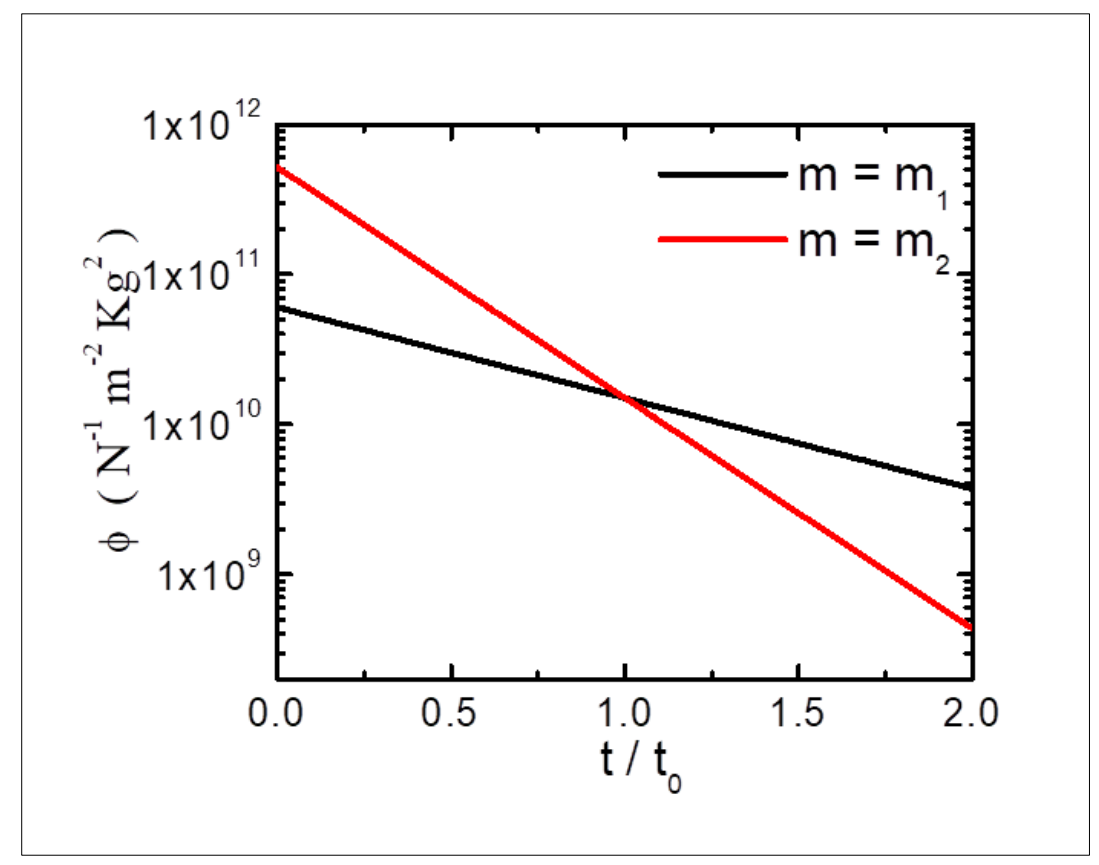

Figure 1 Plots of Brans-Dicke scalar field $(\boldsymbol{\phi})$ versus time for two values of $\boldsymbol{m}$

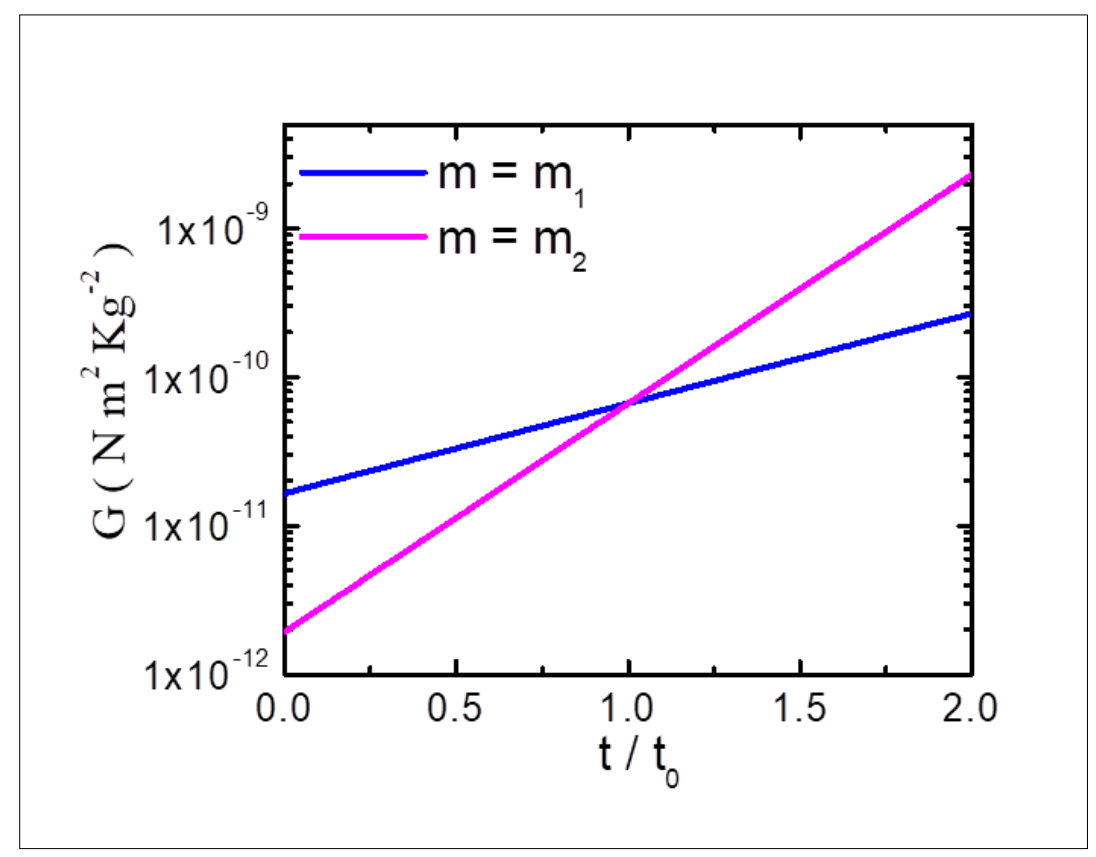

Figure 2 Plots of gravitational constant $(\boldsymbol{G})$ versus time for two values of $\boldsymbol{m}$

\section{Conclusion}

It is certainly of interest to know which of the two values of the parameter $m$ leads to more accurate results. Both values have to be regarded as equally valid unless we have a sufficiently large volume of reasonably accurate experimental data regarding the time variation of the gravitational constant $(G)$. While forming equation (10), on the basis of equation (9), we have assumed that the solution of Brans-Dicke field equations is supposed to lead to exactly those values of the cosmological parameters $\left(H_{0}, q_{0}, \rho_{m_{0}} \& \phi_{0}\right)$ which have been accurately determined from recent astrophysical observations and used for the determination of values of the parameter $m$, based on equation (11). The theoretical validity of the entire formulation depends on the extent of correctness of this assumption. The failure to find a unique value for the parameter $m$ may be a reflection of the fact that the values of the cosmological parameters, obtainable by solving the field equations, are different from their values (listed in section 2) determined from recent astrophysical 
observations and used in the present study. As an extension of this work, one may consider replacing equation (7) by several other ansatzes representing the time variation of the scalar field $(\phi)$, to find the time dependence of the gravitational constant $(G)$ and thereby check the consistency of those findings with those of the present study. An important feature of the present formulation is that it is based on a very simple theoretical concept or plan which has been implemented here through an extremely easy mathematical scheme.

\section{Compliance with ethical standards}

\section{Acknowledgments}

The author is thankful to his institution for the encouragement received from its administration for the continuation of research.

\section{Disclosure of conflict of interest}

There is no conflict of interest associated with this article.

\section{References}

[1] Lu J, Chee G. Cosmology in Poincare gauge gravity with a pseudoscalar torsion. JHEP. 2016; 05(024): 1-26.

[2] Huang QG. An analytic calculation of the growth index for f(R) dark energy model. Eur. Phys. J. C. 2014; 74: 1-5.

[3] Hohmann M, Jarv L, Kuusk P, Randla E, Vilson O. Post-Newtonian parameter $\gamma$ for multiscalar-tensor gravity with a general potential. Phys. Rev. D. 2016; 94: 1-25.

[4] Nojiri S, Odintsov SD, Oikonomou VK. Modified Gravity Theories on a Nutshell: Inflation, Bounce and Late-time Evolution. Phys. Rept. 2017; 692: 1-104.

[5] de la Cruz-Dombriz A, Elizalde E, Odintsov SD, Saez-Gomez D. Spotting deviations from R2 inflation. JCAP. 2016; 05(060): 1-28.

[6] Sotiriou TP. f(R) Gravity and scalar-tensor theory. Class. Quant. Grav. 2006; 23: 5117-5128.

[7] De Felice A, Tsujikawa S. f(R) theories. Living Rev. Relativity. 2010; 13(3): 1-161.

[8] Sotiriou TP, Faraoni V. f(R) Theories of Gravity. Rev. Mod. Phys. 2010; 82: 451-497.

[9] Brans C, Dicke RH. Mach's Principle and a Relativistic Theory of Gravitation. Phys. Rev. 1961; 124: 925-935.

[10] Wang Y, Huang S, Ji Z. Operation management of daily economic dispatch using novel hybrid particle swarm optimization and gravitational search algorithm with hybrid mutation strategy. Mod. Phys. Lett. B. 2017 ; 31 : 1-7.

[11] Abd El-Wahab NH, Salah A. The influence of the classical homogenous gravitational field on interaction of a threelevel atom with a single mode cavity field. Mod. Phys. Lett. B. 2015; 29: 1-11.

[12] Biesiada M, Malec B. A new white dwarf constraint on the rate of change of the gravitational constant. Mon. Not. Roy. Astron. Soc. 2004; 350(2): 644-648.

[13] Benvenuto OG, García-Berro E, Isern J. Asteroseismological bound on $\dot{G} / G$ from pulsating white dwarfs. Phys. Rev. D. 2004; 69: 1-4.

[14] Verbiest JPW, Bailes M, van Straten W, Hobbs GB, Edwards RT, Manchester RN, et al. Precision timing of PSR J0437-4715: an accurate pulsar distance, a high pulsar mass and a limit on the variation of Newton's gravitational constant. Astrophys. J. 2008; 679: 675-680.

[15] Gaztanaga E, Garcia-Berro E, Isern J, Bravo E, Dominguez I. Bounds on the possible evolution of the Gravitational Constant from Cosmological Type-Ia Supernovae. Phys. Rev. D. 2001; 65: 1-9.

[16] Thorsett SE. The Gravitational Constant, the Chandrasekhar Limit, and Neutron Star Masses. Phys. Rev. Lett. 1996; 77(8): 1432-1435.

[17] Bamba K, Momeni D, Myrzakulov R. Kaluza-Klein reduction and Bergmann-Wagoner bi-scalar general action of scalar-tensor gravity. Int. J. Geom. Meth. Mod. Phys. 2015; 12(10): 1-15.

[18] Qiang L, Ma Y, Han M, Yu D. Five-dimensional Brans-Dicke theory and cosmic acceleration. Phys. Rev. D. 2005; 71(6): 1-5. 
[19] Riess AG, Filippenko AV, Challis P, Clocchiatti A, Diercks A, Garnavich PM, et al. Observational Evidence from Supernovae for an Accelerating Universe and a Cosmological Constant. The Astronomical Journal. 1998; 116(3): 1009-1038.

[20] Perlmutter S, Aldering G, Goldhaber G, Knop RA, Nugent P, Castro PG, et al. Measurements of $\Omega$ and $\Lambda$ from 42 High-Redshift Supernovae. The Astrophysical Journal. 1999; 517(2): 565-586.

[21] Spergel DN, Verde L, Peiris HV, Komatsu E, Nolta MR, Bennett CL, et al. First-Year Wilkinson Microwave Anisotropy Probe (WMAP)* Observations: Determination of Cosmological Parameters. Astrophys. J. Suppl. 2003; 148: $175-194$.

[22] Xu LX, Li WB, Lu JB. Holographic dark energy in Brans-Dicke theory. Eur. Phys. J. C. 2009; 60: 135-140.

[23] Weinberg S. Chapter-16 of "Gravitation and Cosmology: Principles and Applications of the General Theory of Relativity". John Wiley \& Sons. 1972.

[24] Roy S, Mukhopadhyay S, Saha A, Chaudhury T. A Study of Some Characteristics of the Accelerated Expansion of the Universe in the Framework of Brans-Dicke Theory. World Scientific News. 2021; 159: 145-166.

[25] Pradhan A, Saha B, Rikhvitsky V. Bianchi type-I transit cosmological models with time dependent gravitational and cosmological constants - re-examined. Indian Journal of Physics. 2015; 89(5): 503-513.

[26] Roy S. Time evolution of the matter content of the expanding universe in the framework of Brans-Dicke gravity. Research in Astronomy and Astrophysics (RAA). 2019; 19(4): 1-14. 